\title{
Existence and uniqueness of fixed point for mixed monotone ternary operators with application
}

\author{
Changchang $\mathrm{Bu}^{1,2}$, Yuqiang Feng ${ }^{1,2^{*}}$ and $\mathrm{Hui} \mathrm{Li}^{1,2}$
}

\section{"Correspondence:}

yqfeng6@126.com

1 School of Science, Wuhan

University of Science and

Technology, Wuhan, 430065,

P.R. China

${ }^{2}$ Hubei Province Key Laboratory of

Systems Science in Metallurgical

Process, Wuhan, 430065, P.R. China

\begin{abstract}
In this paper, partial order theory is used to study the fixed point of a mixed monotone ternary operator $A: P \times P \times P \rightarrow P$. The existence and uniqueness of a fixed point are obtained without assuming the operators to be compact or continuous. In the end, the application to an integral equation is presented. Our results unify, generalize, and complement various known comparable results from the current literature.
\end{abstract}

MSC: $47 \mathrm{H} 05 ; 47 \mathrm{H} 10$

Keywords: fixed point; mixed monotone ternary operator; normal cone

\section{Introduction}

Fixed point theory has fascinated hundreds of researchers since 1922 with the celebrated Banach fixed point theorem. It is well known that mixed monotone operators were introduced by Guo and Lakshmikantham [1] in 1987. Later, Bhaskar and Lakshmikantham [2] introduced the notion of a coupled fixed point and proved some coupled fixed point results under certain conditions, in a complete metric space endowed with a partial order. Their study has not only important theoretical meaning but also wide applications in engineering, nuclear physics, biological chemistry technology, etc. (see [1-8] and the references therein).

Very recently, Harjani et al. [9] have established the existence results of coupled fixed point for mixed monotone operators, and further obtained their applications to integral equations. Berinde and Borcut [10] have introduced the concept of a triple fixed point and proved some related theorems for contractive type operators in partially ordered metric spaces. Zhai [11] has considered mixed monotone operators with convexity and get the existence and uniqueness of a fixed point $(A(u, u)=u$ type) without assuming the operator to be compact or continuous.

Motivated by the work reported in [9-11], the aim of this paper is to discuss the existence and uniqueness of a fixed point $(A(u, u, u)=u$ type) for mixed monotone ternary operators in the context of ordered metric spaces. Our results unify, generalize, and complement various known comparable results from the current literature.

The rest of the paper is organized as follows. In Section 2, we recall some basic definitions and notations which will be used in the sequel. The existence and uniqueness of

(C2014 Bu et al: licensee Springer. This is an Open Access article distributed under the terms of the Creative Commons Attribution License (http://creativecommons.org/licenses/by/2.0), which permits unrestricted use, distribution, and reproduction in any medium, provided the original work is properly cited. 
a fixed point for mixed monotone ternary operators (without assuming the operators to be compact or continuous) are obtained in Section 3. We also present an application in Section 4 to an integral equation to illustrate our results.

\section{Preliminaries}

In this section, we recall some standard definitions and notations needed in the following section. For the convenience of the reader, we suggest that one refers to [1, 2, 10-14] for details.

Throughout this paper, unless otherwise specified, suppose that $(E,\|\cdot\|)$ is a real Banach space which is partially ordered by a cone $P \subset E$, i.e., $x \leq y$ if and only if $y-x \in P$. If $x \leq y$ and $x \neq y$, then we denote $x<y$ or $y>x$. By $\theta$ we denote the zero element of $E$. Recall that a non-empty closed convex set $P \subset E$ is a cone if it satisfies (i) $x \in P, \lambda \geq 0 \Rightarrow \lambda x \in P$; (ii) $x \in P,-x \in P \Rightarrow x=\theta$.

Further, $P$ is called normal if there exists a constant $N>0$ such that, for all $x, y \in E, \theta \leq$ $x \leq y$ implies $\|x\| \leq N\|y\|$; in this case $N$ is called the normality constant of $P$. If $x_{1}, x_{2} \in E$, the set $\left[x_{1}, x_{2}\right]=\left\{x \in E \mid x_{1} \leq x \leq x_{2}\right\}$ is called the order interval between $x_{1}$ and $x_{2}$.

Definition 2.1 (see [10]) $A: P \times P \rightarrow P$ is said to be a mixed monotone operator if $A(x, y)$ is monotone non-decreasing in $x$ and monotone non-increasing in $y$, that is, for any $x, y \in P$,

$$
\begin{array}{lll}
x_{1}, x_{2} \in P, & x_{1} \leq x_{2} \quad \Rightarrow \quad A\left(x_{1}, y\right) \leq A\left(x_{2}, y\right), \\
y_{1}, y_{2} \in P, & y_{1} \leq y_{2} \quad \Rightarrow \quad A\left(x, y_{2}\right) \leq A\left(x, y_{1}\right) .
\end{array}
$$

Definition 2.2 (see [11]) An element $x \in P$ is called a fixed point of $A: P \times P \rightarrow P$ if

$$
A(x, x)=x .
$$

Definition 2.3 (see [10]) $A: P \times P \times P \rightarrow P$ is said to be a mixed monotone operator if $A(x, y, z)$ is monotone non-decreasing in $x, z$ and monotone non-increasing in $y$, that is, for any $x, y, z \in P$

$$
\begin{array}{llll}
x_{1}, x_{2} \in P, & x_{1} \leq x_{2} & \Rightarrow & A\left(x_{1}, y, z\right) \leq A\left(x_{2}, y, z\right), \\
y_{1}, y_{2} \in P, & y_{1} \leq y_{2} \quad \Rightarrow \quad A\left(x, y_{1}, z\right) \geq A\left(x, y_{2}, z\right), \\
z_{1}, z_{2} \in P, & z_{1} \leq z_{2} \quad \Rightarrow & A\left(x, y, z_{1}\right) \leq A\left(x, y, z_{2}\right) .
\end{array}
$$

Definition 2.4 An element $x \in P$ is called a fixed point of $A: P \times P \times P \rightarrow P$ if

$$
A(x, x, x)=x .
$$

\section{Main results}

In this section we consider the existence and uniqueness of a fixed point for mixed monotone ternary operators in ordered Banach spaces. Our first main result is the following.

Theorem 3.1 Let E be a real Banach space and let $P$ be a normal cone in $E . A: P \times P \times P \rightarrow$ $P$ is a mixed monotone ternary operator which satisfies the following: 
$\left(\mathrm{H}_{1}\right)$ for $t \in(0,1), x, y \in P$, there exists $\alpha(t, x, y) \in(1,+\infty)$, such that

$$
A(t x, y, t x) \leq t^{\alpha(t, x, y)} A(x, y, x)
$$

$\left(\mathrm{H}_{2}\right)$ there exist $u_{0}, v_{0}, m_{0} \in P, r \in(0,1)$, such that

$$
\begin{aligned}
& u_{0} \leq r v_{0}, \quad m_{0} \leq r v_{0}, \\
& A\left(u_{0}, v_{0}, m_{0}\right) \geq u_{0}, \quad A\left(v_{0}, u_{0}, v_{0}\right) \leq v_{0}, \quad A\left(m_{0}, v_{0}, u_{0}\right) \geq m_{0}, \\
& A\left(u_{0}, v_{0}, u_{0}\right) \geq u_{0}, \quad A\left(m_{0}, v_{0}, m_{0}\right) \geq m_{0} .
\end{aligned}
$$

Then $A$ has a unique fixed point $u^{*}$ in $\left[u_{0}, r v_{0}\right] \cap\left[m_{0}, r v_{0}\right]$. Moreover, constructing successively the sequences

$$
\begin{aligned}
& x_{n}=A\left(x_{n-1}, y_{n-1}, z_{n-1}\right), \quad y_{n}=A\left(y_{n-1}, x_{n-1}, y_{n-1}\right), \\
& z_{n}=A\left(z_{n-1}, y_{n-1}, x_{n-1}\right), \quad n=1,2, \ldots,
\end{aligned}
$$

for any initial values $x_{0}, y_{0}, z_{0} \in\left[u_{0}, r v_{0}\right] \cap\left[m_{0}, r v_{0}\right]$, we have

$$
\left\|x_{n}-u^{*}\right\| \rightarrow 0, \quad\left\|y_{n}-u^{*}\right\| \rightarrow 0, \quad\left\|z_{n}-u^{*}\right\| \rightarrow 0
$$

as $n \rightarrow \infty$.

Proof Let $w_{0}=r v_{0}, \varepsilon=r^{\alpha\left(r, v_{0}, u_{0}\right)-1}$. Then $w_{0} \geq u_{0}, \varepsilon \in(0,1)$, and

$$
\begin{aligned}
& A\left(w_{0}, u_{0}, w_{0}\right)=A\left(r v_{0}, u_{0}, r v_{0}\right) \leq r^{\alpha\left(r, v_{0}, u_{0}\right)} A\left(v_{0}, u_{0}, v_{0}\right) \\
& \leq r^{\alpha\left(r, v_{0}, u_{0}\right)} v_{0}=r^{\alpha\left(r, v_{0}, u_{0}\right)-1} \cdot r v_{0}=\varepsilon w_{0} \leq w_{0}, \\
& A\left(u_{0}, w_{0}, m_{0}\right)=A\left(u_{0}, r v_{0}, m_{0}\right) \geq A\left(u_{0}, v_{0}, m_{0}\right) \geq u_{0} \text {, } \\
& A\left(m_{0}, w_{0}, u_{0}\right)=A\left(m_{0}, r v_{0}, u_{0}\right) \geq A\left(m_{0}, v_{0}, u_{0}\right) \geq m_{0}
\end{aligned}
$$

Construct successively the sequences

$$
\begin{aligned}
& u_{n}=A\left(u_{n-1}, w_{n-1}, m_{n-1}\right), \quad w_{n}=A\left(w_{n-1}, u_{n-1}, w_{n-1}\right), \quad m_{n}=A\left(m_{n-1}, w_{n-1}, u_{n-1}\right), \\
& w_{n}^{\prime}=\frac{1}{\varepsilon} A\left(w_{n-1}^{\prime}, u_{n-1}, w_{n-1}^{\prime}\right), \quad w_{0}^{\prime}=w_{0}, \quad n=1,2, \ldots
\end{aligned}
$$

From (3.3)-(3.5) and the mixed monotonicity of $A$, we have

$$
\begin{aligned}
& u_{0} \leq u_{1} \leq u_{2} \leq \cdots \leq u_{n} \leq \cdots \leq w_{n} \leq \cdots \leq w_{1} \leq w_{0} \\
& m_{0} \leq m_{1} \leq m_{2} \leq \cdots \leq m_{n} \leq \cdots \leq w_{n} \leq \cdots \leq w_{1} \leq w_{0} .
\end{aligned}
$$

Next we prove that

$$
u_{0} \leq w_{n}^{\prime} \leq w_{0}
$$


From (3.2) and (3.3),

$$
\begin{aligned}
& w_{1}^{\prime}=\frac{1}{\varepsilon} A\left(w_{0}, u_{0}, w_{0}\right) \leq \frac{1}{\varepsilon} \cdot \varepsilon w_{0}=w_{0}, \\
& w_{1}^{\prime}=\frac{1}{\varepsilon} A\left(w_{0}, u_{0}, w_{0}\right) \geq \frac{1}{\varepsilon} A\left(u_{0}, v_{0}, u_{0}\right) \geq \frac{1}{\varepsilon} u_{0} \geq u_{0}, \\
& w_{2}^{\prime}=\frac{1}{\varepsilon} A\left(w_{1}^{\prime}, u_{1}, w_{1}^{\prime}\right) \leq \frac{1}{\varepsilon} A\left(w_{0}, u_{0}, w_{0}\right) \leq \frac{1}{\varepsilon} \cdot \varepsilon w_{0}=w_{0}, \\
& w_{2}^{\prime}=\frac{1}{\varepsilon} A\left(w_{1}^{\prime}, u_{1}, w_{1}^{\prime}\right) \geq \frac{1}{\varepsilon} A\left(u_{0}, v_{0}, u_{0}\right) \geq \frac{1}{\varepsilon} u_{0} \geq u_{0} .
\end{aligned}
$$

Suppose that when $n=k$, we have

$$
u_{0} \leq w_{k}^{\prime} \leq w_{0},
$$

then when $n=k+1$, note that $u_{k} \leq w_{0}=r v_{0} \leq v_{0}$, we obtain

$$
\begin{aligned}
& w_{k+1}^{\prime}=\frac{1}{\varepsilon} A\left(w_{k}^{\prime}, u_{k}, w_{k}^{\prime}\right) \leq \frac{1}{\varepsilon} A\left(w_{0}, u_{0}, w_{0}\right) \leq \frac{1}{\varepsilon} \cdot \varepsilon w_{0}=w_{0}, \\
& w_{k+1}^{\prime}=\frac{1}{\varepsilon} A\left(w_{k}^{\prime}, u_{k}, w_{k}^{\prime}\right) \geq \frac{1}{\varepsilon} A\left(u_{0}, v_{0}, u_{0}\right) \geq \frac{1}{\varepsilon} u_{0} \geq u_{0} .
\end{aligned}
$$

By mathematical induction, we know that (3.8) holds. The same procedure may easily be adapted to obtain

$$
m_{0} \leq w_{n}^{\prime} \leq w_{0}
$$

On the other hand, from (3.1),

$$
\begin{aligned}
w_{1} & =A\left(w_{0}, u_{0}, w_{0}\right)=\varepsilon \frac{1}{\varepsilon} A\left(w_{0}, u_{0}, w_{0}\right)=\varepsilon w_{1}^{\prime}, \\
w_{2} & =A\left(w_{1}, u_{1}, w_{1}\right)=A\left(\varepsilon w_{1}^{\prime}, u_{1}, \varepsilon w_{1}^{\prime}\right) \leq \varepsilon^{\alpha\left(\varepsilon, w_{1}^{\prime}, u_{1}\right)} A\left(w_{1}^{\prime}, u_{1}, w_{1}^{\prime}\right) \\
& =\varepsilon^{\alpha\left(\varepsilon, w_{1}^{\prime}, u_{1}\right)+1} \cdot \frac{1}{\varepsilon} A\left(w_{1}^{\prime}, u_{1}, w_{1}^{\prime}\right) \\
& \leq \varepsilon^{2} w_{2}^{\prime} .
\end{aligned}
$$

Suppose that when $n=k$, we have $w_{k} \leq \varepsilon^{k} w_{k}^{\prime}$. Then when $n=k+1$, in view of (3.1), we obtain

$$
\begin{aligned}
w_{k+1} & =A\left(w_{k}, u_{k}, w_{k}\right) \leq A\left(\varepsilon^{k} w_{k}^{\prime}, u_{k}, \varepsilon^{k} w_{k}^{\prime}\right) \leq\left(\varepsilon^{k}\right)^{\alpha\left(\varepsilon^{k}, w_{k}^{\prime}, u_{k}\right)} A\left(w_{k}^{\prime}, u_{k}, w_{k}^{\prime}\right) \\
& =\varepsilon^{k \alpha\left(\varepsilon^{k}, w_{k}^{\prime}, u_{k}\right)+1} \cdot \frac{1}{\varepsilon} A\left(w_{k}^{\prime}, u_{k}, w_{k}^{\prime}\right) \\
& \leq \varepsilon^{k+1} w_{k+1}^{\prime} .
\end{aligned}
$$

By mathematical induction, we have

$$
w_{n} \leq \varepsilon^{n} w_{n}^{\prime}, \quad n=1,2, \ldots
$$


By (3.6)-(3.10) we get

$$
\begin{aligned}
& \theta \leq w_{n}-u_{n} \leq \varepsilon^{n} w_{n}^{\prime}-u_{n} \leq \varepsilon^{n} w_{n}^{\prime}-\varepsilon^{n} u_{n}=\varepsilon^{n}\left(w_{n}^{\prime}-u_{n}\right) \leq \varepsilon^{n}\left(w_{0}-u_{0}\right), \\
& \theta \leq u_{n+p}-u_{n} \leq w_{n}-u_{n}, \quad \theta \leq w_{n}-w_{n+p} \leq w_{n}-u_{n} ; \\
& \theta \leq w_{n}-m_{n} \leq \varepsilon^{n} w_{n}^{\prime}-m_{n} \leq \varepsilon^{n} w_{n}^{\prime}-\varepsilon^{n} m_{n}=\varepsilon^{n}\left(w_{n}^{\prime}-m_{n}\right) \leq \varepsilon^{n}\left(w_{0}-m_{0}\right), \\
& \theta \leq m_{n+p}-m_{n} \leq w_{n}-m_{n} .
\end{aligned}
$$

Noting that $P$ is normal and $\varepsilon \in(0,1)$, we have

$$
\begin{aligned}
& \left\|w_{n}-u_{n}\right\| \leq N \varepsilon^{n}\left\|w_{0}-u_{0}\right\| \rightarrow 0 \quad(\text { as } n \rightarrow \infty), \\
& \left\|w_{n}-m_{n}\right\| \leq N \varepsilon^{n}\left\|w_{0}-m_{0}\right\| \rightarrow 0 \quad(\text { as } n \rightarrow \infty) .
\end{aligned}
$$

Further,

$$
\begin{aligned}
& \left\|u_{n+p}-u_{n}\right\| \leq N\left\|w_{n}-u_{n}\right\| \rightarrow 0 \quad(\text { as } n \rightarrow \infty), \\
& \left\|w_{n}-w_{n+p}\right\| \leq N\left\|w_{n}-u_{n}\right\| \rightarrow 0 \quad(\text { as } n \rightarrow \infty), \\
& \left\|m_{n+p}-m_{n}\right\| \leq N\left\|w_{n}-m_{n}\right\| \rightarrow 0 \quad(\text { as } n \rightarrow \infty) .
\end{aligned}
$$

Here $N$ is the normality constant.

So, we can claim that $\left\{u_{n}\right\},\left\{w_{n}\right\}$, and $\left\{m_{n}\right\}$ are Cauchy sequences. Since $E$ is complete, there exist $u^{*}, w^{*}, m^{*} \in P$ such that

$$
u_{n} \rightarrow u^{*}, \quad w_{n} \rightarrow w^{*}, \quad m_{n} \rightarrow m^{*} \quad(\text { as } n \rightarrow \infty) .
$$

By (3.6), (3.7), respectively, we know that

$$
\begin{aligned}
& u_{0} \leq u_{n} \leq u^{*} \leq w^{*} \leq w_{n} \leq w_{0}, \\
& m_{0} \leq m_{n} \leq m^{*} \leq w^{*} \leq w_{n} \leq w_{0},
\end{aligned}
$$

and then

$$
\begin{aligned}
& \theta \leq w^{*}-u^{*} \leq w_{n}-u_{n} \leq \varepsilon^{n}\left(w_{0}-u_{0}\right), \\
& \theta \leq w^{*}-m^{*} \leq w_{n}-m_{n} \leq \varepsilon^{n}\left(w_{0}-m_{0}\right) .
\end{aligned}
$$

Further, $\left\|w^{*}-u^{*}\right\| \leq N \varepsilon^{n}\left\|w_{0}-u_{0}\right\| \rightarrow 0$ (as $n \rightarrow \infty$ ), and thus $w^{*}=u^{*}$. Similarly, we get $\left\|w^{*}-m^{*}\right\| \leq N \varepsilon^{n}\left\|w_{0}-m_{0}\right\| \rightarrow 0($ as $n \rightarrow \infty)$, and thus $w^{*}=m^{*}$. Consequently, $w^{*}=u^{*}=$ $m^{*}$. Then we obtain

$$
u_{n+1}=A\left(u_{n}, w_{n}, m_{n}\right) \leq A\left(u^{*}, u^{*}, u^{*}\right) \leq A\left(w_{n}, u_{n}, w_{n}\right)=w_{n+1} .
$$

Letting $n \rightarrow \infty$, then we get

$$
A\left(u^{*}, u^{*}, u^{*}\right)=u^{*} .
$$

That is, $u^{*}$ is a fixed point of $A$ in $\left[u_{0}, r v_{0}\right] \cap\left[m_{0}, r v_{0}\right]$. 
In the following, we prove that $u^{*}$ is the unique fixed point of $A$ in $\left[u_{0}, r v_{0}\right] \cap\left[m_{0}, r v_{0}\right]$. Suppose that there exists $x^{*} \in\left[u_{0}, r v_{0}\right] \cap\left[m_{0}, r v_{0}\right]$ such that $A\left(x^{*}, x^{*}, x^{*}\right)=x^{*}$. Then $u_{0} \leq$ $x^{*} \leq w_{0}$ and $m_{0} \leq x^{*} \leq w_{0}$. By mathematical induction and the mixed monotonicity of $A$, we have

$$
u_{n+1}=A\left(u_{n}, w_{n}, m_{n}\right) \leq x^{*}=A\left(x^{*}, x^{*}, x^{*}\right) \leq A\left(w_{n}, u_{n}, w_{n}\right)=w_{n+1} .
$$

Then from the normality of $P$, we have $x^{*}=u^{*}$.

Moreover, constructing successively the sequences

$$
\begin{aligned}
& x_{n}=A\left(x_{n-1}, y_{n-1}, z_{n-1}\right), \quad y_{n}=A\left(y_{n-1}, x_{n-1}, y_{n-1}\right), \\
& z_{n}=A\left(z_{n-1}, y_{n-1}, x_{n-1}\right), \quad n=1,2, \ldots,
\end{aligned}
$$

for any initial values $x_{0}, y_{0}, z_{0} \in\left[u_{0}, r v_{0}\right] \cap\left[m_{0}, r v_{0}\right]$, we have $u_{n} \leq x_{n}, w_{n} \geq y_{n}, m_{n} \leq z_{n}$, $n=1,2, \ldots$. Letting $n \rightarrow \infty$ yields $x_{n} \rightarrow u^{*}, y_{n} \rightarrow u^{*}, z_{n} \rightarrow u^{*}$ as $n \rightarrow \infty$.

Remark 3.1 It is evident from (3.1) that for $t \in(0,1), x, y \in P$, there exists $\alpha\left(t, \frac{1}{t} x, y\right) \in$ $(1,+\infty)$, such that

$$
A\left(\frac{1}{t} x, y, \frac{1}{t} x\right) \geq \frac{1}{t^{\alpha\left(t, \frac{1}{t} x, y\right)}} A(x, y, x)
$$

Remark 3.2 Let $\alpha(t, x, y)$ be a constant $\alpha \in(1,+\infty)$, then Theorem 3.1 also holds.

Corollary 3.2 Let E be a real Banach space and let $P$ be a normal cone in $E$. A:P $\times P \times P \rightarrow$ $P$ is a mixed monotone ternary operator which satisfies $\left(\mathrm{H}_{2}\right)$ and, for $t \in(0,1), x, y \in P$, there exists $\alpha \in(1,+\infty)$, such that $A(t x, y, t x) \leq t^{\alpha} A(x, y, x)$. Then $A$ has a unique fixed point $u^{*}$ in $\left[u_{0}, r v_{0}\right] \cap\left[m_{0}, r v_{0}\right]$. Moreover, constructing successively the sequences

$$
\begin{aligned}
& x_{n}=A\left(x_{n-1}, y_{n-1}, z_{n-1}\right), \quad y_{n}=A\left(y_{n-1}, x_{n-1}, y_{n-1}\right), \\
& z_{n}=A\left(z_{n-1}, y_{n-1}, x_{n-1}\right), \quad n=1,2, \ldots,
\end{aligned}
$$

for any initial values $x_{0}, y_{0}, z_{0} \in\left[u_{0}, r v_{0}\right] \cap\left[m_{0}, r v_{0}\right]$, we have $u_{n} \leq x_{n}, w_{n} \geq y_{n}, m_{n} \leq z_{n}$, $n=1,2, \ldots$. Letting $n \rightarrow \infty$ yields $x_{n} \rightarrow u^{*}, y_{n} \rightarrow u^{*}, z_{n} \rightarrow u^{*}$ as $n \rightarrow \infty$.

Following the lines of the proof of Theorem 3.1, we obtain an immediate consequence.

Corollary 3.3 (see [11]) Let E be a real Banach space and let $P$ be a normal cone in $E$. $A: P \times P \rightarrow P$ is a mixed monotone operator which satisfies the following:

$\left(\mathrm{H}_{3}\right)$ for $t \in(0,1), x, y \in P$, there exists $\alpha(t, x, y) \in(1,+\infty)$, such that

$$
A(t x, y) \leq t^{\alpha(t, x, y)} A(x, y)
$$

$\left(\mathrm{H}_{4}\right)$ there exist $u_{0}, v_{0} \in P, r \in(0,1)$, such that

$$
u_{0} \leq r v_{0}, \quad A\left(u_{0}, v_{0}\right) \geq u_{0}, \quad A\left(v_{0}, u_{0}\right) \leq v_{0} \text {. }
$$


Then $A$ has a unique fixed point $u^{*}$ in $\left[u_{0}, r v_{0}\right]$. Moreover, constructing successively the sequences

$$
x_{n}=A\left(x_{n-1}, y_{n-1}\right), \quad y_{n}=A\left(y_{n-1}, x_{n-1}\right), \quad n=1,2, \ldots,
$$

for any initial values $x_{0}, y_{0} \in\left[u_{0}, r v_{0}\right]$, we have

$$
\left\|x_{n}-u^{*}\right\| \rightarrow 0, \quad\left\|y_{n}-u^{*}\right\| \rightarrow 0
$$

as $n \rightarrow \infty$.

Theorem 3.4 Let E be a real Banach space and let $P$ be a normal cone in $E . A: P \times P \times P \rightarrow$ $P$ is a mixed monotone ternary operator which satisfies (3.1) and

$\left(\mathrm{H}_{5}\right)$ for $R \in(1,+\infty), x, y, z \in P$ there exist $\alpha\left(\frac{1}{R}, R x, y, z\right), \alpha\left(\frac{1}{R}, x, y, R z\right) \in(1,+\infty)$ such that

$$
\begin{aligned}
& A(R x, y, z) \geq R^{\alpha\left(\frac{1}{R}, R x, y, z\right)} A(x, y, z), \\
& A(x, y, R z) \geq R^{\alpha\left(\frac{1}{R}, x, y, R z\right)} A(x, y, z) ;
\end{aligned}
$$

$\left(\mathrm{H}_{6}\right)$ there exist $u_{0}, v_{0}, m_{0} \in P, R \in(1,+\infty)$, such that

$$
\begin{aligned}
& v_{0} \geq R u_{0}, \quad v_{0} \geq R m_{0}, \\
& A\left(u_{0}, v_{0}, m_{0}\right) \geq u_{0}, \quad A\left(v_{0}, u_{0}, v_{0}\right) \leq v_{0}, \quad A\left(m_{0}, v_{0}, u_{0}\right) \geq m_{0}, \\
& A\left(u_{0}, v_{0}, u_{0}\right) \geq u_{0}, \quad A\left(m_{0}, v_{0}, m_{0}\right) \geq m_{0} .
\end{aligned}
$$

Then the operator equation $A(w, w, w)=b w$ has a unique solution $w^{*}$ in $\left[R u_{0}, v_{0}\right] \cap$ $\left[R m_{0}, v_{0}\right]$, where $b=\min \left\{R^{\alpha\left(\frac{1}{R}, R u_{0}, v_{0}, m_{0}\right)-1}, R^{\alpha\left(\frac{1}{R}, m_{0}, v_{0}, R u_{0}\right)-1}\right\}$. Moreover, constructing successively the sequences

$$
\begin{aligned}
& x_{n}=b^{-1} A\left(x_{n-1}, y_{n-1}, z_{n-1}\right), \quad y_{n}=b^{-1} A\left(y_{n-1}, x_{n-1}, y_{n-1}\right), \\
& z_{n}=b^{-1} A\left(z_{n-1}, y_{n-1}, x_{n-1}\right), \quad n=1,2, \ldots,
\end{aligned}
$$

for any initial values $x_{0}, y_{0}, z_{0} \in\left[R u_{0}, v_{0}\right] \cap\left[R m_{0}, v_{0}\right]$, we have

$$
\left\|x_{n}-w^{*}\right\| \rightarrow 0, \quad\left\|y_{n}-w^{*}\right\| \rightarrow 0, \quad\left\|z_{n}-w^{*}\right\| \rightarrow 0
$$

as $n \rightarrow \infty$.

Remark 3.3 Two comments with respect to conditions (3.12) and (3.13) are in order:

(a) A sufficient condition on $A$ for (3.12) to be satisfied is that for $t \in(0,1), x, y, z \in P$, there exists $\alpha(t, x, y, z) \in(1,+\infty)$, such that

$$
A(t x, y, z) \leq t^{\alpha(t, x, y, z)} A(x, y, z) .
$$

(b) A sufficient condition on $A$ for (3.13) to be satisfied is that for $t \in(0,1), x, y, z \in P$, there exists $\alpha(t, x, y, z) \in(1,+\infty)$, such that

$$
A(x, y, t z) \leq t^{\alpha(t, x, y, z)} A(x, y, z) .
$$


Proof of Theorem 3.4 Let $w_{0}=R u_{0}$. Then $v_{0} \geq w_{0}$. Note that $b>1$, from (3.12)-(3.14),

$$
\begin{aligned}
A\left(w_{0}, v_{0}, m_{0}\right) & =A\left(R u_{0}, v_{0}, m_{0}\right) \geq R^{\alpha\left(\frac{1}{R}, R u_{0}, v_{0}, m_{0}\right)} A\left(u_{0}, v_{0}, m_{0}\right) \\
& =R^{\alpha\left(\frac{1}{R}, R u_{0}, v_{0}, m_{0}\right)-1} R A\left(u_{0}, v_{0}, m_{0}\right) \geq b R u_{0}=b w_{0} \geq w_{0}, \\
A\left(v_{0}, w_{0}, v_{0}\right) & =A\left(v_{0}, R u_{0}, v_{0}\right) \leq A\left(v_{0}, u_{0}, v_{0}\right) \leq v_{0}, \\
A\left(m_{0}, v_{0}, w_{0}\right) & =A\left(m_{0}, v_{0}, R u_{0}\right) \geq R^{\alpha\left(\frac{1}{R}, m_{0}, v_{0}, R u_{0}\right)} A\left(m_{0}, v_{0}, u_{0}\right) \\
& =R^{\alpha\left(\frac{1}{R}, m_{0}, v_{0}, R u_{0}\right)-1} R A\left(m_{0}, v_{0}, u_{0}\right) \geq b A\left(m_{0}, v_{0}, u_{0}\right) \geq b m_{0} \geq m_{0} .
\end{aligned}
$$

Set $B(x, y, z)=b^{-1} A(x, y, z), x, y, z \in P$. Then from the above inequalities, we have

$$
\begin{aligned}
& B\left(w_{0}, v_{0}, m_{0}\right)=b^{-1} A\left(w_{0}, v_{0}, m_{0}\right) \geq b^{-1} b w_{0}=w_{0}, \\
& B\left(v_{0}, w_{0}, v_{0}\right)=b^{-1} A\left(v_{0}, w_{0}, v_{0}\right) \leq b^{-1} v_{0} \leq v_{0}, \\
& B\left(m_{0}, v_{0}, w_{0}\right)=b^{-1} A\left(m_{0}, v_{0}, w_{0}\right) \geq b^{-1} b m_{0}=m_{0} .
\end{aligned}
$$

Also, construct successively the sequences

$$
\begin{array}{ll}
w_{n}=B\left(w_{n-1}, v_{n-1}, m_{n-1}\right), & v_{n}=B\left(v_{n-1}, w_{n-1}, v_{n-1}\right), \quad m_{n}=B\left(m_{n-1}, v_{n-1}, w_{n-1}\right), \\
v_{n}^{\prime}=b B\left(v_{n-1}^{\prime}, w_{n-1}, v_{n-1}^{\prime}\right), & v_{0}^{\prime}=v_{0}, \quad n=1,2, \ldots
\end{array}
$$

From (3.18) and the mixed monotonicity of $A$, we have

$$
\begin{aligned}
& w_{0} \leq w_{1} \leq w_{2} \leq \cdots \leq w_{n} \leq \cdots \leq v_{n} \leq \cdots \leq v_{1} \leq v_{0} \\
& m_{0} \leq m_{1} \leq m_{2} \leq \cdots \leq m_{n} \leq \cdots \leq v_{n} \leq \cdots \leq v_{1} \leq v_{0}
\end{aligned}
$$

Next we prove that

$$
w_{0} \leq v_{n}^{\prime} \leq v_{0}, \quad n=1,2, \ldots
$$

By (3.11) and (3.14), we have

$$
\begin{aligned}
A\left(w_{0}, v_{0}, w_{0}\right) & =A\left(R u_{0}, v_{0}, R u_{0}\right) \geq R^{\alpha\left(\frac{1}{R}, R u_{0}, v_{0}\right)} A\left(u_{0}, v_{0}, u_{0}\right) \\
& \geq R A\left(u_{0}, v_{0}, u_{0}\right) \\
& \geq R u_{0} \\
& =w_{0} .
\end{aligned}
$$

From (3.15)-(3.17) and (3.22),

$$
\begin{aligned}
& v_{1}^{\prime}=b B\left(v_{0}^{\prime}, w_{0}, v_{0}^{\prime}\right)=b B\left(v_{0}, w_{0}, v_{0}\right)=A\left(v_{0}, w_{0}, v_{0}\right) \leq v_{0}, \\
& v_{1}^{\prime}=b B\left(v_{0}^{\prime}, w_{0}, v_{0}^{\prime}\right)=b B\left(v_{0}, w_{0}, v_{0}\right) \geq b B\left(w_{0}, v_{0}, m_{0}\right)=A\left(w_{0}, v_{0}, m_{0}\right) \geq w_{0}, \\
& v_{2}^{\prime}=b B\left(v_{1}^{\prime}, w_{1}, v_{1}^{\prime}\right) \leq b B\left(v_{0}, w_{0}, v_{0}\right)=A\left(v_{0}, w_{0}, v_{0}\right) \leq v_{0}, \\
& v_{2}^{\prime}=b B\left(v_{1}^{\prime}, w_{1}, v_{1}^{\prime}\right) \geq b B\left(w_{0}, v_{0}, w_{0}\right)=A\left(w_{0}, v_{0}, w_{0}\right)=A\left(R u_{0}, v_{0}, R u_{0}\right) \geq w_{0} .
\end{aligned}
$$


Suppose that when $n=k$, we have

$$
w_{0} \leq v_{k}^{\prime} \leq v_{0},
$$

then when $n=k+1$, recalling (3.16) and (3.22), we obtain

$$
\begin{aligned}
& v_{k+1}^{\prime}=b B\left(v_{k}^{\prime}, w_{k}, v_{k}^{\prime}\right) \leq b B\left(v_{0}, w_{0}, v_{0}\right)=A\left(v_{0}, w_{0}, v_{0}\right) \leq v_{0}, \\
& v_{k+1}^{\prime}=b B\left(v_{k}^{\prime}, w_{k}, v_{k}^{\prime}\right) \geq b B\left(w_{0}, v_{0}, w_{0}\right)=A\left(w_{0}, v_{0}, w_{0}\right)=A\left(R u_{0}, v_{0}, R u_{0}\right) \geq w_{0} .
\end{aligned}
$$

By mathematical induction, we know that (3.21) holds. The same procedure may easily be adapted to obtain

$$
m_{0} \leq v_{n}^{\prime} \leq v_{0}, \quad n=1,2, \ldots
$$

On the other hand, from (3.1),

$$
\begin{aligned}
v_{1} & =B\left(v_{0}, w_{0}, v_{0}\right)=\frac{1}{b} b B\left(v_{0}, w_{0}, v_{0}\right)=\frac{1}{b} b B\left(v_{0}^{\prime}, w_{0}, v_{0}^{\prime}\right)=\frac{1}{b} v_{1}^{\prime}, \\
v_{2} & =B\left(v_{1}, w_{1}, v_{1}\right)=B\left(\frac{1}{b} v_{1}^{\prime}, w_{1}, \frac{1}{b} v_{1}^{\prime}\right) \leq\left(\frac{1}{b}\right)^{\alpha\left(\frac{1}{b}, v_{1}^{\prime}, w_{1}\right)} B\left(v_{1}^{\prime}, w_{1}, v_{1}^{\prime}\right) \\
& =\left(\frac{1}{b}\right)^{\alpha\left(\frac{1}{b}, v_{1}^{\prime}, w_{1}\right)+1} b B\left(v_{1}^{\prime}, w_{1}, v_{1}^{\prime}\right) \leq\left(\frac{1}{b}\right)^{2} v_{2}^{\prime} .
\end{aligned}
$$

Suppose that when $n=k$, we have $v_{k} \leq\left(\frac{1}{b}\right)^{k} v_{k}^{\prime}$. Then when $n=k+1$, in view of (3.1), we obtain

$$
\begin{aligned}
v_{k+1} & =B\left(v_{k}, w_{k}, v_{k}\right) \leq B\left(\left(\frac{1}{b}\right)^{k} v_{k}^{\prime}, w_{k},\left(\frac{1}{b}\right)^{k} v_{k}^{\prime}\right) \\
& \leq\left(\left(\frac{1}{b}\right)^{k}\right)^{\alpha\left(\left(\frac{1}{b}\right)^{k}, v_{k}^{\prime}, w_{k}\right)} B\left(v_{k}^{\prime}, w_{k}, v_{k}^{\prime}\right) \\
& \leq\left(\frac{1}{b}\right)^{k \alpha\left(\left(\frac{1}{b}\right)^{k}, v_{k}^{\prime}, w_{k}\right)+1} b B\left(v_{k}^{\prime}, w_{k}, v_{k}^{\prime}\right) \\
& \leq\left(\frac{1}{b}\right)^{k+1} v_{k+1}^{\prime} .
\end{aligned}
$$

By mathematical induction, we have

$$
v_{n} \leq\left(\frac{1}{b}\right)^{n} v_{n}^{\prime}, \quad n=1,2, \ldots
$$

By (3.19)-(3.24) we get

$$
\begin{aligned}
\theta & \leq v_{n}-w_{n} \leq\left(\frac{1}{b}\right)^{n} v_{n}^{\prime}-w_{n} \leq\left(\frac{1}{b}\right)^{n} v_{n}^{\prime}-\left(\frac{1}{b}\right)^{n} w_{n} \\
& =\left(\frac{1}{b}\right)^{n}\left(v_{n}^{\prime}-w_{n}\right) \leq\left(\frac{1}{b}\right)^{n}\left(v_{0}-w_{0}\right)
\end{aligned}
$$




$$
\begin{aligned}
\theta & \leq w_{n+p}-w_{n} \leq v_{n}-w_{n}, \quad \theta \leq v_{n}-v_{n+p} \leq v_{n}-w_{n} \\
\theta & \leq v_{n}-m_{n} \leq\left(\frac{1}{b}\right)^{n} v_{n}^{\prime}-m_{n} \leq\left(\frac{1}{b}\right)^{n} v_{n}^{\prime}-\left(\frac{1}{b}\right)^{n} m_{n} \\
& =\left(\frac{1}{b}\right)^{n}\left(v_{n}^{\prime}-m_{n}\right) \leq\left(\frac{1}{b}\right)^{n}\left(v_{0}-m_{0}\right), \\
\theta & \leq m_{n+p}-m_{n} \leq v_{n}-m_{n}, \quad \theta \leq v_{n}-v_{n+p} \leq v_{n}-m_{n} .
\end{aligned}
$$

Note that $P$ is normal and $b>1$, we have

$$
\begin{aligned}
& \left\|v_{n}-w_{n}\right\| \leq N\left(\frac{1}{b}\right)^{n}\left\|v_{0}-w_{0}\right\| \rightarrow 0 \quad(\text { as } n \rightarrow \infty), \\
& \left\|v_{n}-m_{n}\right\| \leq N\left(\frac{1}{b}\right)^{n}\left\|v_{0}-m_{0}\right\| \rightarrow 0 \quad(\text { as } n \rightarrow \infty) .
\end{aligned}
$$

Further,

$$
\begin{aligned}
& \left\|w_{n+p}-w_{n}\right\| \leq N\left\|v_{n}-w_{n}\right\| \rightarrow 0 \quad(\text { as } n \rightarrow \infty), \\
& \left\|v_{n}-v_{n+p}\right\| \leq N\left\|v_{n}-w_{n}\right\| \rightarrow 0 \quad(\text { as } n \rightarrow \infty), \\
& \left\|m_{n+p}-m_{n}\right\| \leq N\left\|v_{n}-m_{n}\right\| \rightarrow 0 \quad(\text { as } n \rightarrow \infty) .
\end{aligned}
$$

Here $N$ is the normality constant.

So, we can claim that $\left\{w_{n}\right\},\left\{v_{n}\right\}$, and $\left\{m_{n}\right\}$ are Cauchy sequences. Since $E$ is complete, there exist $w^{*}, v^{*}, m^{*} \in P$ such that

$$
w_{n} \rightarrow w^{*}, \quad v_{n} \rightarrow v^{*}, \quad m_{n} \rightarrow m^{*} \quad(\text { as } n \rightarrow \infty) .
$$

By (3.19), (3.20), respectively, we know that

$$
\begin{aligned}
& w_{0} \leq w_{n} \leq w^{*} \leq v^{*} \leq v_{n} \leq v_{0}, \\
& m_{0} \leq m_{n} \leq m^{*} \leq v^{*} \leq v_{n} \leq v_{0},
\end{aligned}
$$

and then

$$
\begin{aligned}
& \theta \leq v^{*}-w^{*} \leq v_{n}-w_{n} \leq\left(\frac{1}{b}\right)^{n}\left(v_{0}-w_{0}\right), \\
& \theta \leq v^{*}-m^{*} \leq v_{n}-m_{n} \leq\left(\frac{1}{b}\right)^{n}\left(v_{0}-m_{0}\right) .
\end{aligned}
$$

Further, $\left\|v^{*}-w^{*}\right\| \leq N\left(\frac{1}{b}\right)^{n}\left\|v_{0}-w_{0}\right\| \rightarrow 0$ (as $\left.n \rightarrow \infty\right)$, and thus $v^{*}=w^{*}$. Similarly, we get $\left\|v^{*}-m^{*}\right\| \leq N\left(\frac{1}{b}\right)^{n}\left\|v_{0}-m_{0}\right\| \rightarrow 0$ (as $\left.n \rightarrow \infty\right)$, and thus $v^{*}=m^{*}$. Consequently, $w^{*}=v^{*}=$ $m^{*}$. Then we obtain

$$
w_{n+1}=B\left(w_{n}, v_{n}, m_{n}\right) \leq B\left(w^{*}, w^{*}, w^{*}\right) \leq B\left(v_{n}, w_{n}, v_{n}\right)=v_{n+1} \text {. }
$$

Letting $n \rightarrow \infty$, we get

$$
B\left(w^{*}, w^{*}, w^{*}\right)=w^{*} .
$$


That is, the operator equation $A(w, w, w)=b w$ has a unique solution $w^{*}$ in $\left[R u_{0}, v_{0}\right] \cap$ $\left[R m_{0}, v_{0}\right]$.

In the following, we prove that $w^{*}$ is the unique solution of $A(w, w, w)=b w$ in $\left[R u_{0}, v_{0}\right] \cap$ $\left[R m_{0}, v_{0}\right]$. Suppose that there exists $x^{*} \in\left[R u_{0}, v_{0}\right] \cap\left[R m_{0}, v_{0}\right]$ such that $A\left(x^{*}, x^{*}, x^{*}\right)=b x^{*}$. Then $w_{0} \leq x^{*} \leq v_{0}$ and $m_{0} \leq x^{*} \leq v_{0}$. By mathematical induction and the mixed monotonicity of $A$, we have

$$
w_{n+1}=B\left(w_{n}, v_{n}, m_{n}\right) \leq x^{*}=B\left(x^{*}, x^{*}, x^{*}\right) \leq B\left(v_{n}, w_{n}, v_{n}\right)=v_{n+1} \text {. }
$$

Then from the normality of $P$, we have $x^{*}=w^{*}$.

Moreover, constructing successively the sequences

$$
\begin{aligned}
& x_{n}=b^{-1} A\left(x_{n-1}, y_{n-1}, z_{n-1}\right), \quad y_{n}=b^{-1} A\left(y_{n-1}, x_{n-1}, y_{n-1}\right), \\
& z_{n}=b^{-1} A\left(z_{n-1}, y_{n-1}, x_{n-1}\right), \quad n=1,2, \ldots,
\end{aligned}
$$

for any initial values $x_{0}, y_{0}, z_{0} \in\left[R u_{0}, v_{0}\right] \cap\left[R m_{0}, v_{0}\right]$, we have $w_{n} \leq x_{n}, v_{n} \geq y_{n}, m_{n} \leq z_{n}$, $n=1,2, \ldots$. Letting $n \rightarrow \infty$ yields $x_{n} \rightarrow w^{*}, y_{n} \rightarrow w^{*}, z_{n} \rightarrow w^{*}$ as $n \rightarrow \infty$.

From the proof of Theorem 3.4, we can easily obtain the following conclusion.

Corollary 3.5 (see [11]) Let E be a real Banach space and let $P$ be a normal cone in $E$. $A: P \times P \rightarrow P$ is a mixed monotone operator which satisfies $\left(\mathrm{H}_{3}\right)$ and

$\left(\mathrm{H}_{7}\right)$ there exist $u_{0}, v_{0} \in P, R \in(1,+\infty)$ such that

$$
v_{0} \geq R u_{0}, \quad A\left(u_{0}, v_{0}\right) \geq u_{0}, \quad A\left(v_{0}, u_{0}\right) \leq v_{0} .
$$

Then the operator equation $A(w, w)=b w$ has a unique solution $w^{*}$ in $\left[R u_{0}, v_{0}\right]$, where $b=$ $R^{\alpha\left(\frac{1}{R}, R u_{0}, v_{0}\right)-1}$. Moreover, constructing successively the sequences

$$
x_{n}=b^{-1} A\left(x_{n-1}, y_{n-1}\right), \quad y_{n}=b^{-1} A\left(y_{n-1}, x_{n-1}\right), \quad n=1,2, \ldots,
$$

for any initial values $x_{0}, y_{0} \in\left[R u_{0}, v_{0}\right]$, we have

$$
\left\|x_{n}-w^{*}\right\| \rightarrow 0, \quad\left\|y_{n}-w^{*}\right\| \rightarrow 0
$$

as $n \rightarrow \infty$.

\section{Application}

As application of our results, we investigate the solvability of the following integral equation:

$$
x(\tau)=\int_{0}^{1} k(\tau, s)\left[\frac{x^{\alpha_{1}}(s)}{1+x^{\alpha_{2}}(s)}+x^{\alpha_{3}}(s)\right] \mathrm{d} s
$$

with $\alpha_{1}, \alpha_{3}>1, \alpha_{2}>0$.

Put $E=C[0,1]$ (the space of continuous functions defined on [0,1] endowed with supremum norm). Let $P=\{x \in E \mid x(t) \geq 0, \forall t \in[0,1]\}$, then $E$ is a Banach space and $P$ is a normal cone. Suppose that $k(\tau, s):[0,1] \times[0,1] \rightarrow R^{++}\left(R^{++}\right.$denotes the positive real numbers $)$ 
is continuous and $0<\int_{0}^{1} k(\tau, s) \mathrm{d} s \leq \frac{1}{2}$. In the following, we prove that (4.1) has a unique solution.

Consider the integral operator $A: P \times P \times P \rightarrow P$ defined by

$$
A(u, v, m)=\int_{0}^{1} k(\tau, s)\left[\frac{u^{\alpha_{1}}(s)}{1+v^{\alpha_{2}}(s)}+m^{\alpha_{3}}(s)\right] \mathrm{d} s
$$

with $\alpha_{1}, \alpha_{3}>1, \alpha_{2}>0$.

It is clear that $A(u, v, m)$ is a mixed monotone ternary operator. We shall show that $A(u, v, m)$ satisfies $\left(\mathrm{H}_{2}\right)$ and for $t \in(0,1), x, y \in P$, there exists $\alpha \in(1,+\infty)$, such that $A(t x, y, t x) \leq t^{\alpha} A(x, y, x)$.

In fact, let $u_{0}(\tau) \equiv 0, m_{0}(\tau) \equiv 0, v_{0}(\tau) \equiv 1$, then

$$
\begin{aligned}
& A\left(u_{0}, v_{0}, u_{0}\right)=0 \geq u_{0}, \\
& A\left(v_{0}, u_{0}, v_{0}\right)=2 \int_{0}^{1} k(\tau, s) \mathrm{d} s \leq 1 .
\end{aligned}
$$

On the other hand, noting that for any $t \in(0,1)$, letting

$$
\alpha=\min \left\{\alpha_{1}, \alpha_{3}\right\}>1
$$

we obtain

$$
\begin{aligned}
A(t x, y, t x) & =\int_{0}^{1} k(\tau, s)\left[\frac{t^{\alpha_{1}} x^{\alpha_{1}}(s)}{1+y^{\alpha_{2}}(s)}+t^{\alpha_{3}} x^{\alpha_{3}}(s)\right] \mathrm{d} s \\
& \leq \int_{0}^{1} k(\tau, s)\left[\frac{t^{\alpha} x^{\alpha_{1}}(s)}{1+y^{\alpha_{2}}(s)}+t^{\alpha} x^{\alpha_{3}}(s)\right] \mathrm{d} s \\
& =t^{\alpha} \int_{0}^{1} k(\tau, s)\left[\frac{x^{\alpha_{1}}(s)}{1+y^{\alpha_{2}}(s)}+x^{\alpha_{3}}(s)\right] \mathrm{d} s \\
& =t^{\alpha} A(x, y, x) .
\end{aligned}
$$

Hence, all the hypotheses of Corollary 3.2 are satisfied. The operator

$$
A(u, v, m)=\int_{0}^{1} k(\tau, s)\left[\frac{u^{\alpha_{1}}(s)}{1+v^{\alpha_{2}}(s)}+m^{\alpha_{3}}(s)\right] \mathrm{d} s
$$

has a unique fixed point in $\left[u_{0}, v_{0}\right]$, i.e., the integral equation (4.1) has a unique solution in $\left[u_{0}, v_{0}\right]$.

\section{Competing interests}

The authors declare that they have no competing interests.

\section{Authors' contributions}

All authors contributed equally to the writing of this paper. All authors read and approved the final manuscript.

\section{Acknowledgements}

This research is supported by the Doctoral Fund of Education Ministry of China (20134219120003), the Natural Science Foundation of Hubei Province (2013CFA131), the National Natural Science Foundation of China (71231007) and Hubei Province Key Laboratory of Systems Science in Metallurgical Process (z201302). 


\section{References}

1. Guo, D, Lakskmikantham, V: Coupled fixed points of nonlinear operators with applications. Nonlinear Anal. 11(5), 623-632 (1987)

2. Bhaskar, TG, Lakskmikantham, V: Fixed point theory in partially ordered metric spaces and applications. Nonlinear Anal. 65, 1379-1393 (2006)

3. Guo, D: Existence and uniqueness of positive fixed point for mixed monotone operators with applications. Appl. Anal. 46, 91-100 (1992)

4. Zhang, Z: New fixed point theorems of mixed monotone operators and applications. J. Math. Anal. Appl. 204 307-319 (1996)

5. Zhang, S, Ma, Y: Coupled fixed points for mixed monotone condensing operators and an existence theorem of the solution for a class of functional equations arising in dynamic programming. J. Math. Anal. Appl. 160, 468-479 (1991)

6. Sun, Y: A fixed point theorem for mixed monotone operator with applications. J. Math. Anal. Appl. 156, 240-252 (1991)

7. Lian, $X, L i$, Y: Fixed point theorems for a class of mixed monotone operators with applications. Nonlinear Anal. 67, 2752-2762 (2007)

8. $\mathrm{Wu}, \mathrm{Y}, \mathrm{Liang}, \mathrm{Z}$ : Existence and uniqueness of fixed points for mixed monotone operators with applications. Nonlinear Anal. 65, 1913-1924 (2006)

9. Harjani, J, Lopez, B, Sadarangani, K: Fixed point theorems for mixed monotone operators and applications to integral equations. Nonlinear Anal. 74, 1749-1760 (2011)

10. Berinde, $V$, Borcut, M: Tripled fixed point theorems for contractive type mappings in partially ordered metric spaces. Nonlinear Anal. 74(15), 4889-4897 (2011)

11. Zhai, C: Fixed point theorems for a class of mixed monotone operators with convexity. Fixed Point Theory Appl. 2013 $119(2013)$

12. Lei, $\mathrm{P}, \mathrm{Lin}, \mathrm{X}$, Jiang, D: Existence and uniqueness of solutions for singular nonlinear elliptic boundary value problems. Nonlinear Anal. 69, 2773-2779 (2008)

13. Guo, D, Lakskmikantham, V: Nonlinear Problems in Abstract Cones. Academic Press, New York (1998)

14. Zhai, CB, Guo, CM: On $\alpha$-convex operators. J. Math. Anal. Appl. 316, 556-565 (2006)

10.1186/1687-1812-2014-223

Cite this article as: Bu et al.: Existence and uniqueness of fixed point for mixed monotone ternary operators with application. Fixed Point Theory and Applications 2014, 2014:223

\section{Submit your manuscript to a SpringerOpen ${ }^{\circ}$ journal and benefit from:}

- Convenient online submission

- Rigorous peer review

- Immediate publication on acceptance

- Open access: articles freely available online

- High visibility within the field

- Retaining the copyright to your article 